\title{
ФУНКЦИОНАЛЬНАЯ МИТРАЛЬНАЯ РЕГУРГИТАЦИЯ ПРИ АНЕВРИЗМЕ ВОСХОДЯЩЕГО ОТДЕЛА АОРТЫ У БОЛЬНЫХ ПОДВЕРГАЮЩИХСЯ ОПЕРАЦИИ НА АОРТАЛЬНОМ КЛАПАНЕ
}

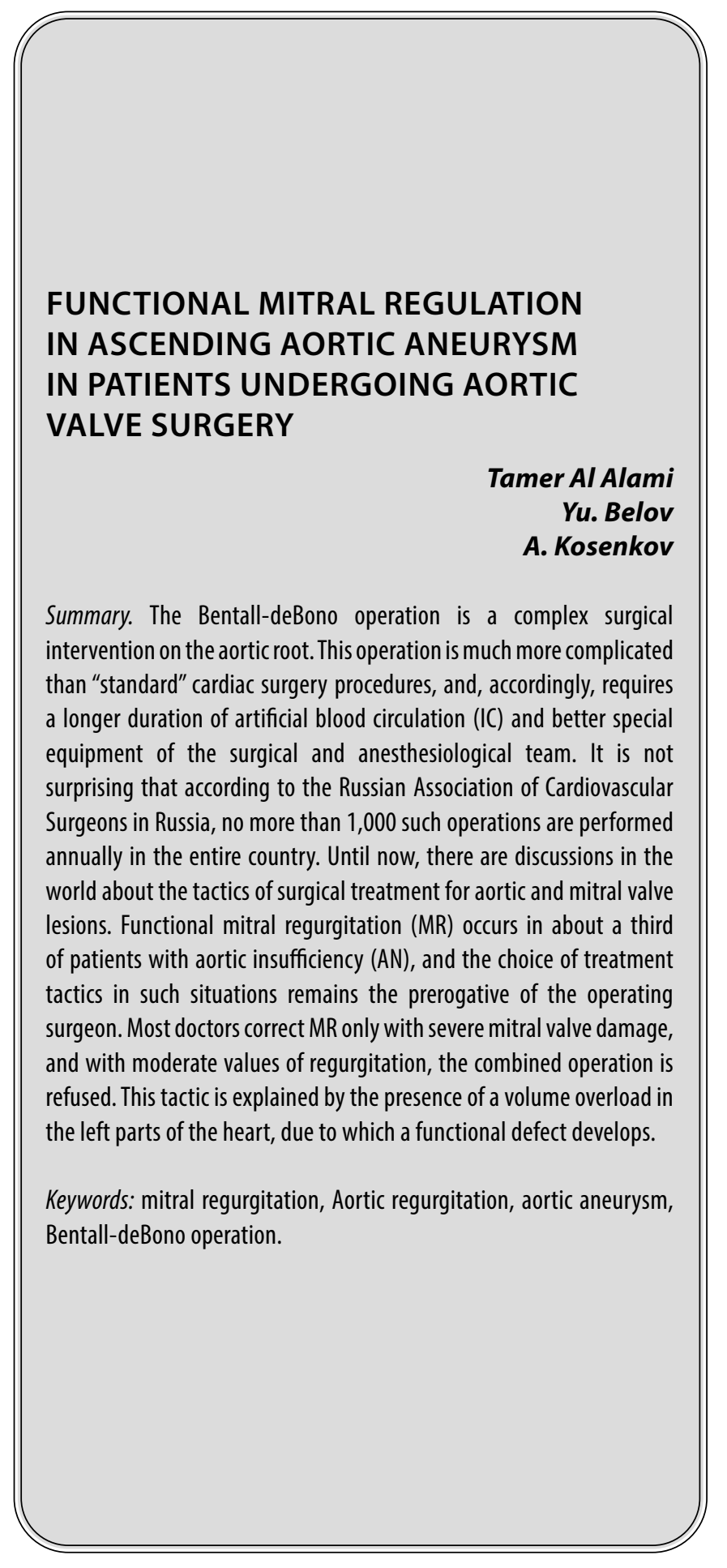

Тамер Аль Алами

Аспирант, Первый Московский Государственный Медицинский Университет им. И.М. Сеченова Минздрава России (Сеченовский Университет)

r.alami@doctor.com

Белов Юрий Владимирович

Д.м.н., профессор, Академик РАН, Первый Московский Государственный Медицинский Университет им. И.М. Сеченова (Сеченовский Университет) belov@sechenov.ru

Косенков Александр Николаевич

Д.м.н., профессор, н.с., Первый Московский Государственный Медицинский Университет им. И.М. Сеченова Минздрава России (Сеченовский Университет)

kosenkov@sechenov.ru

Аннотация. Операция Bentall-deBono является сложным хирургическим вмешательством на корне аорты. Данная операция значительно сложнее «стандартных» кардиохирургических процедур, а соответственно, требует большей длительности искусственного кровообращения (ИК) и лучшего специального оснащения хирургической и анестезиологической бригады. Не удивительно, что по данным Российской ассоциации сердечно-сосудистых хирургов в России ежегодно выполняется не более 1000 таких операций во всей стране. До сих пор в мире ведутся дискуссии о тактике хирургического лечения при поражении аортального и митрального клапанов. Функциональная митральная регургитация (МР) встречается примерно у трети больных с аортальной недостаточности (АН) и выбор тактики лечения в таких ситуациях остается прерогативой оперирующего хирурга. Большинство врачей корригируют МР только при тяжелом поражении митрального клапана, а при умеренных значениях регургитации от сочетанной операции отказываются. Такую тактику объясняют наличием объемной перегрузки в левых отделах сердца за счет чего развивается функциональный порок.

Ключевые слова: митральная регургитация, Аортальная недостаточность, аневризма аорты, Операция Bentall-deBono. 


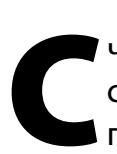
читается, что после коррекции аортальной недостаточности (АН) перегрузка самостоятельно регрессирует за счет обратного ремоделирования левого желудочка (ЛЖ) и вместе с ней нормализуется кровоток через митральный клапан [3, с. 741]. Кроме того, коррекция двух клапанных пороков является более травматичной процедурой, летальность при которой достигает 12\% [4, с. 547]. В современной литературе вопрос о влиянии функциональной МР на послеоперационное течение больных и отдаленные результаты освещен в небольшом количестве исследований [5, с. 62]. Причем в данных работах нет четкого разделения на группы по причине порока.

При анализе литературы нам удалось найти всего 2 исследования, которые изучали отдаленные результаты при «чистой» функциональной МР (без возможных влияний на структуру митрального клапана различных заболеваний) [6, с. 32]. В обоих исследованиях авторы не отметили увеличение госпитальной летальности при двухклапанной коррекции. С другой стороны, в исследовании других автором отмечено, что наличие МР является предиктором неудовлетворительных результатов после изолированной коррекции AK [7, c. 1443]. В мета-анализе, проведенном Harling L et al. [8, c7 1087]. продемонстрировано, что отдаленная выживаемость в сроках 3 и 5 лет при наличии МР значительной хуже, чем при отсутствии данной порока у больных. Необходимо отметить, что данное исследование проводили при изучении больных со стенозом AK, и, вероятно, не может быть в абсолютной степени перенесено на модель больных с AH. Barreiro CJ et al. [9, с. 1443]. описывают свои данные по неудовлетворительным результатам преимущественно у больных с ишемической MP, a Ruel et al. [10, с. 1541]. в смешенном исследовании АН и аортального стеноза получили данные об отсутствии влияния МР не прогноз. Ряд исследований так же склоняются к мысли, что МР не является предрасполагающим фактором при стабильном течении хронической сердечной недостаточности (XСH), но объем левого предсердия более 5см, легочная гипертензия, наличие постоянной формы фибрилляции предсердий (ФП) может являться предиктором неудовлетворительных отдаленных результатов [11, с. 32]. Необходимо отметить, что у большинства больных после изолированной коррекции порока АК в раннем послеоперационном периоде уменьшается размер левого предсердия [12, с. 1378]. Именно на этот факт и делают акцент большинство хирургов, демонстрируя раннее обратное ремоделирование сердца после изолированной операции.

Чтобы понять концепцию большинства хирургов необходимо уточнить особенности функциональной МР. На сегодняшний день считается, что функциональная
МР является вторичным изменением после развития дисфункции ЛЖ. Она возникает в результате нарушения взаимосвязи между сокращением Лж и закрытием створок митрального клапана [13, с. 745]. Данные изменения встречаются в ряде ситуаций: перегрузка левых отделов сердца на фоне существующего порока аортального клапана, пролапс митрального клапана, симметричная или ассиметричная дилатация фиброзного кольца аортального клапана и при нарушении целостности папилярных мышц [14, с. 1826]. Снижение систолической функции ЛЖ и нетипичная анатомия левого желудочка так же могут являться причиной формирования MP[15, с. 1594].

Исходя из этого был сделан вывод о первичности поражения ЛЖ при развитии данного порока. В связи с исследованиями на большой группе больных, где был доказан неблагоприятный прогноз у больных с функциональной МР при наличии других тяжелых заболевания сердца был сделан вывод о необходимости разработки алгоритма по определению тяжести порока и вкладе его в общее нарушение гемодинамики.

При установлении вклада порока в качество жизни больных после изолированных хирургических вмешательств необходим адекватный подход к выбору тактики лечени. В одном из исследований была попытка оспорить общепринятую теорию. Beaudoin et al. провели ретроспективный анализ у 816 больных с умеренной АН и МР. Несмотря на гипотезу авторов им не удалось полноценно оспорит постулат: “митральная регургитация в первую очередь является заболеванием левого желудочка, а затем клапана сердца". По данным эхокардиографического исследования диастолический объем лж был в полтора раза больше, в контрольной группе.

Ряд полученных результатов, например об увеличении площади створок клапана на фоне имеющейся АН согласуются с другими исследованиями.

Именно эту особенность авторы возводят как "основное доказательство" отдельной болезни клапана. Однако, увеличение створок может являться компенсаторным механизмом для предотвращения перегрузки сердца. Так же следует помнить об анатомии митрального клапана, в котором зона кооптации не соответствует краю створки. На фоне дилатации ЛЖ происходит удлинение створок митрального клапана и зона коаптации некоторое время может смещаться к краю створки. Как только анатомической длинны створки не хватает - возникает регургитация. Если рассматривать типичные митральные пороки, например болезнь Барлоу, то при гистологических исследованиях отмечено увеличение коллагена с утолщением створки за счет этого развиваются адаптационные механизмы, 
данная особенность отмечена так же у больных без соединительнотканной дисплазии.

АН, как правило, приводит к постепенной дилатации Лж на протяжении жизни человека с данным пороком. За счет затяжного течения данного заболевания у митрального клапана остается достаточно времени для развития адаптационных механизмов. При уже сформированной MP у больных наступает этап "быстрого" прогрессирования левожелудочковй дисфункции за счет перегрузки левых отделов сердца. Так же возможно, что обратный поток крови из АК способствует растяжению створок и фиброзного кольца митрального клапана (МК), однако данная концепция требует уточнения для выбора адекватной тактики лечения таких больных.

Как мы уже говорили ранее, наиболее целесообразно изучать вопросы функционального поражения митрального клапана в модели левожелудочковой дисфункции. В работе Rossi A. et al было продемонстрировано как в результате прогрессирования аортального порока происходила дилатация Лж и смещение папилярных мышц. В ответ на это смещается точка кооптации и развивается порок, данный механизм схож по своему развитию с ишемической кардиомиопатией. В другом исследовании было продемонстрировано, что на прогрессирование МР так же оказывает роль сократимость левого предсердия и наличие фибрилляции предсердий.

В эхокардиографических работах, проведенных на модели изолированного протезирования аортального клапана (ПАК) было получено множество значимых для понимания процесса ремоделирования данных. При 3D анализе была продемонстрирована изменчивость непосредственно структур митрального клапана после ПАК.

Диаметр фиброзного кольца и длина створок МК уменьшились после ПАК в среднем на 16,3\%. Изменение геометрии фиброзного кольца МК происходило преимущественно за счет сокращения передней полуокружности. На фоне изменения геометрии уменьшалась степень регургитации, оцениваемая по площади vena contracta, что так же подтверждено в ряде других исследований. Кроме патофизиологических особенностей данного состояния необходимо рассматривать и клиническую значимость функциональной МР. В ряде публикаций авторы пришли к выводу, что наличие МР до коррекции порока клапана сердца несет в себе повышенный риск развития осложнений как в ближайшем, так и в отдаленном периоде после операции. Еще в 1898 году Simpson IA et al в своей работе утверждали, что полуколичественные методы оценки МР не могут быть эффективными для каких-либо заключений относительно значимости или вклада митрального порока в сердечную дисфункцию. Только в 2010 году Европейское общество эхокардиографии утвердило данный постулат, рекомендовав использовать количественную оценку МР через измерение эффективной площади регургитации. До сих пор большинство исследователей не проводят оценку по количественным методам, поэтому результаты большинства исследований не могут быть доказаны с учетом методом современного статистического анализа. Несмотря на «слабую» оценку непосредственно митрального клапана сердца, ряд закономерностей так называемой «митрализации» аортального порока было найдено.

Важной закономерностью при отсутствии коррекции МР у больных оперированных на АК является повышенные риски развития фибрилляции предсердий, прогрессирования сердечной недостаточности и отека легких. Несмотря на то, что МР в настоящее время достаточно хорошо поддается медикаментозному лечению до сих пор не установлены показания и ее устранению при необходимости операции на АК.

При исходном стенозе АК снижение степени функциональной МР наблюдали у 60-80\% больных, но для АН однозначных цифр до сих пор не получено.

ЭХО-кардиографические данные ряда исследований демонстрируют повышение фракции выброса (ФВ), снижение конечнодиастолического объема (КДО) ЛЖ, снижение степени митральной и трикуспидальной регургитации, а так же давления в легочной артерии в течение первых недель после операции. Однако часть представленных индексов могут изменяться за счет влияния анестезиологических препаратов и травмирующих факторов операции, поэтому адекватная оценка должна проводиться не менее чем через 6 месяцев.

Ruel M. et al. провели наблюдательное исследование за динамикой степени MP у больных после коррекции АН. Через 18 месяцев после успешной операции были оценены гемодинамические характеристики больных. Оказалось, что вне зависимости от наличия до операции МР у 10,6\% развивается регургитация 2 степени, а при уже имеющейся до операции патологии результаты зависят от исходного КДР ЛЖ. Если у больного КДР более 45 мм, то значимая МР остается у 42,1\% больных, а если это значение меньше - всего у $20,0 \%$.

В другом исследовании было продемонстрировано, что изолированное ПАК у больных с МР 3+ степенью ведет к снижению 5-летней выживаемости с 70\% до 43\% в сравнении с группой больных, у которых МР была меньшей степени. В этом же исследовании пока- 
зано, что при одномоментной коррекции МР отдаленная выживаемость составляет 70\% при пластической реконструкции и $60 \%$ при протезировании клапана.

Больных с синдромом Марфана следует рассматривать как отдельную категорию. Учитывая выраженное генетически-обусловленное поражение соединительной ткани у этих больных МР встречается в 28-91\% случаев. Данная особенность связана с микосматозной дегенерацией створок МК и дестабилизации межклеточного матрикса в соединительной ткани всех органов. В исследовании, посвященном изучению МР у больных после реконструкции корня аорты было сделано заключения о малой частоте потребности в одномоментной коррекции МК. Критерием необходимости реконструкции авторы считают МР 2-й степени, она встречалась у $9 \%$ больных, что соответствовало частоте больных без генерализованного поражения соединительной ткани. В тоже время, авторы докладывают, что у 7\% больных без реконструктивной операции на МК степень регургитации увеличивается в послеоперационном периоде.
Одномоментная коррекция МР приводит к улучшению сопоставления створок МК. При выполнении реконструктивной операции на митральном клапане происходит сокращение эффективной площади отверстия на 40-50\%. Такое уменьшение диаметра фиброзного кольца МК приводит к увеличению площади зоны коаптации, тем самым увеличивая “физиологический резерв" МК и подклапанных структур, предотвращая дальнейшие риски дилатации фиброзного кольца. За счет изолированной анулопластики в сочетании с коррекцией порока АК можно добиться достаточного эффекта для “физиологической” и длительно существующей коррекции MP.

Исходя из описанных выше механизмов возможно предположить, что при коррекции АН при наличии выраженной функциональной МР достаточно выполнить изолированную аннулопластику. Эта процедура значимо не увеличит травматичность операции, но создаст физиологически обоснованную коаптацию стврок митрального клапана.

\section{ЛИТЕРАТУРА}

1. Бакерия Л.А., Ступаков И.Н., Гудкова Р.Г., и др. Хирургическое лечение болезней системы кровообращения в Российской Федерации (2010-2014). Вестник Росздравнадзора № 12016.

2. Sabbah HN, Rosman H, Kono T, Alam M, Khaja F, Goldstein S. On the mechanism of secondary mitral regurgitation. Am J Cardiol 1993;72: 1074-6., Moazami $\mathrm{N}$, diodato MD, Moon MR, Lawton JS, Pasque MK, Herren RL et al. Does secondary mitral regurgitation improve with isolated aortic valve replacement? J Card Surg 2004.

3. Harris MH, Malenka DJ, Haney MF, Jayne JE, Hettleman B, Plehn JF et al. Improvement in mitral regurgitation after aortic valve replacement. Am J Cardiol 1997.

4. Rankin JS, Hammill BG, Ferguson JTB, Glower DD, O'Brien SM, DeLong ER et al. Determinants of operative mortality in valvular heart surgery. JThorac Cardiovasc Surg 2006.

5. Wan CKN, Suri RM, Li Z, Orsulak TA, Daly RC, Schaff HV et al. Management of moderate secondary mitral regurgitation at the time of aortic valve replacement: is concomitant mitral valve repair necessary? J Thorac Cardiovasc Surg 2009;137:635-40, Ruel M, Kapila V, Price J, Kulik A, Burwash IG, Mesana TG. Natural history and predictors of outcome in patients with concomitant second- ary mitral regurgitation at the time of aortic valve replacement. Circulation 2006;114(Suppl I): 1541-6, Caballero-Borrego J, Gómez-Doblas JJ, Cabrera-Bueno F, García-Pinilla JM, Melero JM, Porras C et al. Incidence, associated factors and evolu- tion of non-severe secondary mitral regurgitation in patients with severe aortic stenosis undergoing aortic valve replacement. Eur J Cardiothorac Surg 2008.

6. Gillinov MA, Blackstone EH, Cosgrove DM, White J, Kerr P, Marullo A et al. Mitral valve repair with aortic valve replacement is superior to double valve replacement. J Thorac Cardiovasc Surg 2003;125:1372-87, Coutinho GF, Correia PM, Pancas R, Antunes MJ. Management of moderate secondary mitral regurgitation at the time of aortic valve surgery. Eur J Cardiothorac Surg. 2013.

7. Barreiro CJ, Patel ND, Fitton TP, Williams JA, Bonde PN, Chan V et al. Aortic valve replacement and concomitant mitral valve regurgitation in the elderly: impact on survival and secondary outcome. Circulation 2005.

8. Harling L, Saso S, Jarral Oa, Kourliouros A, Kidher E, Athanasiou T. Aortic valve replacement for aortic stenosis in patients with concomitant mitral regurgitation: should the mitral valve be dealt with? Eur J Cardiothorac Surg 2011.

9. Barreiro CJ, Patel ND, Fitton TP, Williams JA, Bonde PN, Chan V et al. Aortic valve replacement and concomitant mitral valve regurgitation in the elderly: impact on survival and secondary outcome. Circulation 2005.

10. Ruel M, Kapila V, Price J, Kulik A, Burwash IG, Mesana TG. Natural history and predictors of outcome in patients with concomitant second-ary mitral regurgitation at the time of aortic valve replacement. Circulation 2006.

11. Coutinho GF, Correia PM, Pancas R, Antunes MJ. Management of moderate secondary mitral regurgitation at the time of aortic valve surgery. Eur J Cardiothorac Surg. 2013.

12. Unger P, Plein D, Camp GV, Cosyns B, Pasquet A, Henrad V et al. Effects of valve replacement for aortic stenosis on mitral regurgitation. Am J Cardiol 2008.

13. Levine RA, Schwammenthal E. Ischemic mitral regurgitation on the threshold of a solution: from paradoxes to unifying concepts. Circu- lation 2005.

14. Kwan J, Shiota T, Agler DA, et al. Geometric differences of the mitral apparatus between ischemic and dilated cardiomyopathy with significant mitral regurgitation. Real-time three-dimensional echocardiography study. Circulation 2003;107:1135-40, He S, Fontaine AA, Schwammenthal E, Yoganathan AP, 
Levine RA. Integrated mechanism for functional mitral regurgitation: leaflet re- striction versus coapting force: in vitro studies. Circulation 1997;96: 182634, Otsuji Y, Handschumacher MD, Schwammenthal E, et al. Insights from three-dimensional echocardiography into the mechanism of functional mitral regurgitation: direct in vivo demonstration of altered leaflet tethering geometry. Circulation 1997.

15. Kaul S, Pearlman JD, Touchstone DA, Esquival L. Prevalence and mechanisms of mitral regurgitation in the absence of intrinsic abnor- malities of the mitral leaflets. Am Heart J 1989;118:763-72, Kono T, Sabbah HN, Rosman H, Alam M, Jafri S, Goldstein S. Left ventricular shape is the primary determinant of functional mitral regurgitation in heart failure. J Am Coll Cardiol 1992.

( ) Тамер Аль Алами ( r.alami@doctor.com ),

Белов Юрий Владимирович ( belov@sechenov.ru ), Косенков Александр Николаевич ( kosenkov@sechenov.ru ).

Журнал «Современная наука: актуальные проблемы теории и практики»

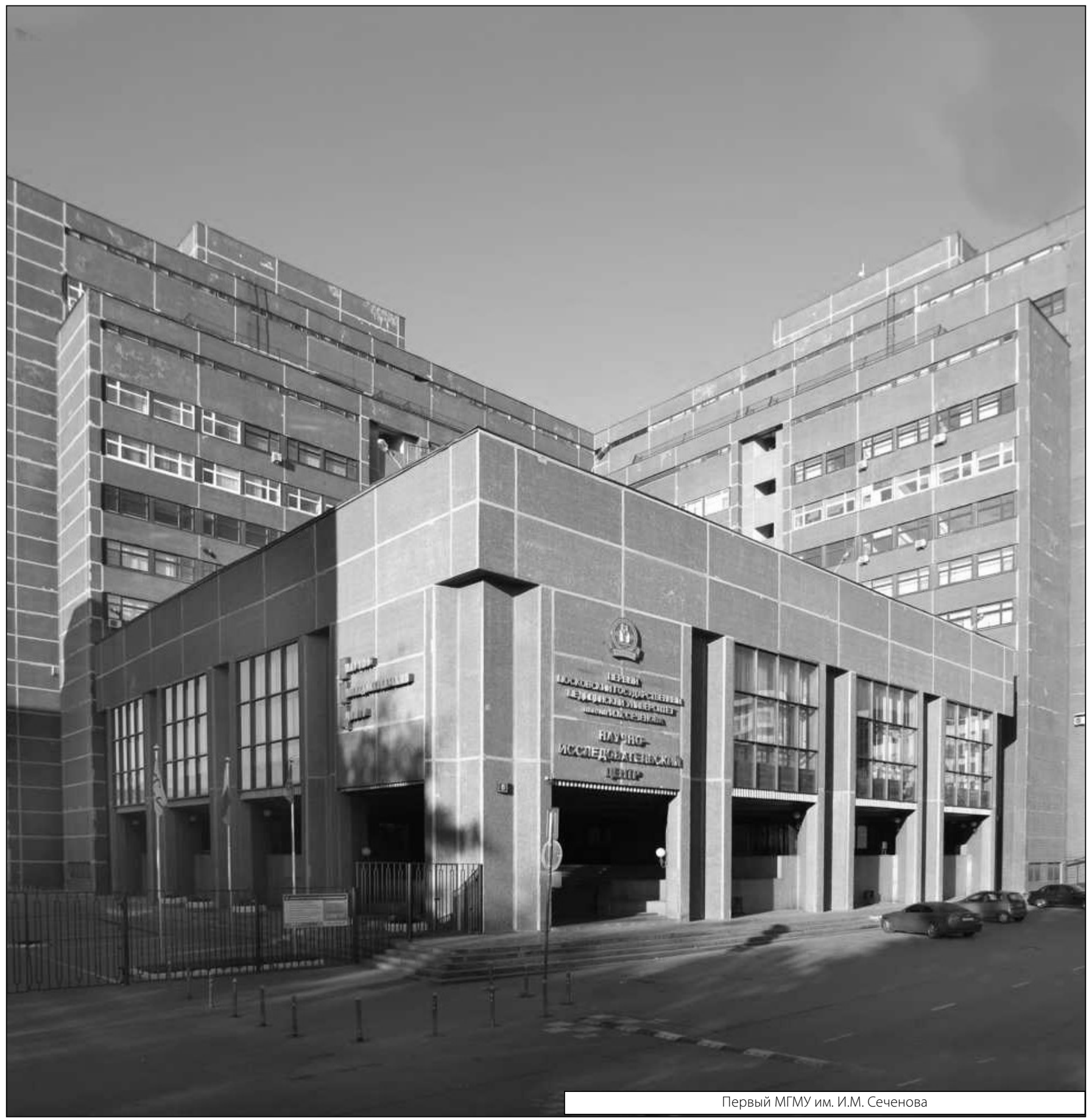

